\title{
Modeling of Optical and IR Spectra of $M$ and Brown Dwarfs
}

\author{
Yakiv V. Pavlenko \\ Main Astronomical Observatory of National Academy of Sciences, \\ Golosiiv woods, Kyiv-127, 03680 Ukraine
}

\begin{abstract}
Procedure and results of computation of optical and infrared spectra of ultracool $\left(1000<\mathrm{T}_{\text {eff }}<3000 \mathrm{~K}\right)$ objects are discussed. LTE synthetic spectra and spectral energy distributions in wide spectral region $\lambda \lambda$ 0.6-10 microns) are computed for model atmospheres from grids of different authors taking into account opacities provided by water and other molecules.

Essential interest will be paid for problems of realization of lithium and deuterium tests which can be used for assessment of objects of substellar masses and big planets and refining of scenarios of evolution of young stars and substellar objects.
\end{abstract}

\section{M Dwarfs: Lithium Test}

The "lithium test" (Rebolo et al. 1992) has been widely used to determine the true substellar nature of several brown dwarfs (Rebolo et al. 1996, Ruiz et al. 1997, Martín et al. 1998). Additionally the frontier of objects which burn lithium and those less massive which have not yet depleted it (Lithium Depletion Boundary, LDB) provide an alternative method to date clusters younger than 150 Myr (Martín et al. 1998; Stauffer et al. 1998, 1999; Basri \& Martín, 1999).

A few factors affect the Li I line formation in M-dwarf atmospheres:

- Blending by molecular lines. In fact we can work with pseudoequivalent widths of lines (Pavlenko 1998, Zapatero Osorio et al. 2002).

- Depletion into the molecular species: $\mathrm{LiCl}, \mathrm{LiOH}, \mathrm{LiF}$ in low temperature regions (Pavlenko et al. 1995).

In Fig.1 we show some fits to observed Li I lines in spectrum of young brown dwarf Teide1 (Rebolo et al. 1996). Computations were carried out for model atmospheres from NEXTGEN grid of Hauschildt et al. (1999), TiO (Plez 1998) and VALD (Kupka 1999) line lists using WITA6 program (Pavlenko 2000). We adopt dissociation potential $\mathrm{D}_{0}=7.92 \mathrm{eV}$ for $\mathrm{TiO}$, chemical equilibrium was computed for a mixture of $\sim 100$ species. Computed for microturbulent velocity $V_{t}=2 \mathrm{~km} / \mathrm{s}$ synthetic spectra were convolved with gaussian of $0.2 \mathrm{~nm}$. The best fits provide $\log \mathrm{N}(\mathrm{Li})=3.3 \mathrm{in}$ atmosphere of Teide1. However, fit of theoretical spectrum to the observed $\mathrm{TiO}$ band head at $672 \mathrm{~nm}$ is rather poor - perhaps, NEXGEN models do not describe properly a structure of the outermost layers of Teide1's atmosphere. 

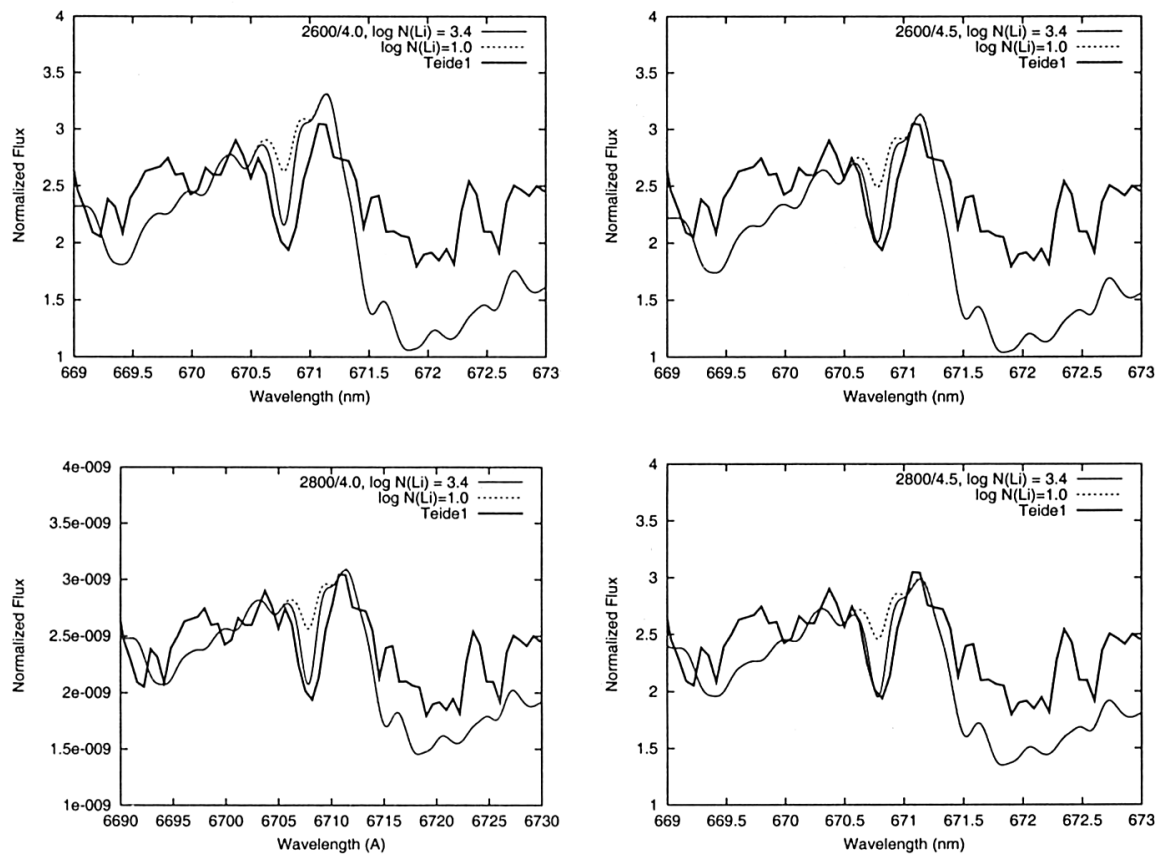

Figure 1. Fits to Li I $670.8 \mathrm{~nm}$ line of Teide1.

\section{L and T Dwarfs: Deuterium Test}

Deuterium is an element which can be depleted at lower temperatures than Lithium $\left(\mathrm{T} \sim 810^{5} \mathrm{~K}\right)$ in the fusion reaction $\mathrm{D}\left(\mathrm{p},{ }^{3} \mathrm{He}\right)$. That means that objects below a mass around 0.011-0.013 $M_{\odot}$ (Saumon et al. 1996, Burrows et al. 1997, Chabrier et al. 2000) should preserve their deuterium from the time of formation. Those objects will never burn any element and several authors established at this frontier the separation between brown dwarfs and planets (Saumon et al. 1996, Béjar et al. 1999).

In Fig.2 we show some spectral energy distributions of ultracool dwarf of $\mathrm{T}_{\text {eff }} 1200 \mathrm{~K}, \log \mathrm{g}=5.0$ from a grid of "dusty" C-models of Tsuji (1999) and Partridge \& Schwenke (1997) list of $\mathrm{H}_{2} \mathrm{O}$ and HDO lines. Chemical equilibrium of $\mathrm{HDO}$ and $\mathrm{H}_{2} \mathrm{O}$ were computed using information of molecular levels of $\mathrm{H}_{2} \mathrm{O}$ in AMES database (Partridge \& Schwenke 1997). Synthetical spectra were computed by program WITA6 with step $0.05 \mathrm{~nm}$ and then convolved with gaussian of $1 \mathrm{~nm}$. SEDs were computed for different D/O ratios (see Pavlenko 2002 for more details).

Note:

- Bands of $\mathrm{HDO}$ are shifted in the IR region in respect to $\mathrm{H}_{2} \mathrm{O}$ bands.

- The best regions for $\mathrm{D} / \mathrm{H}$ ratio determination are $3.5-4,6-7$ microns as well as a region around 8 micron. 


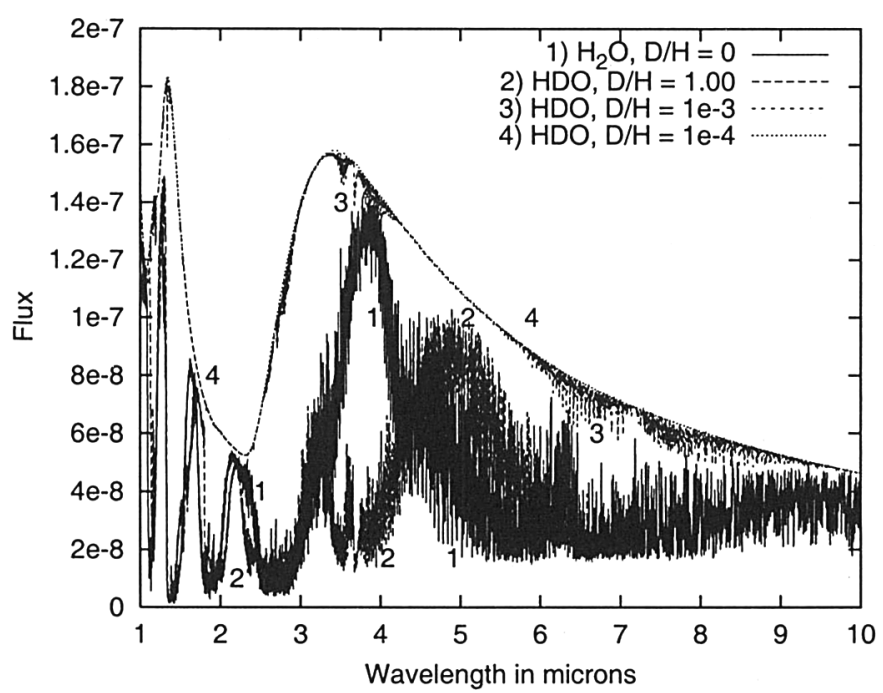

Figure 2. $\mathrm{HDO}$ and $\mathrm{H}_{2} \mathrm{O}$ molecular bands computed for different $\mathrm{D} / \mathrm{H}$ for $2800 / 5.0$ and $1200 / 5.0$ model atmospheres.

A comparison of computed spectra which were convolved with a gaussian of $0.1 \mathrm{~nm}$ (i.e., it corresponds to a resolution of $\mathrm{R} \sim 30000$ ). A detection of HDO lines in the spectra of coolest dwarfs seems to be a challenge for largest telescopes and best observers.

Acknowledgments. I thank the SOC and LOC of the Symposium and our Chairman Prof. Eduardo Martín for financial support of my participation. I thank Dr. María Rosa Zapatero Osorio for providing the Teide1's spectrum in digital form, Prof. T. Tsuji for his "dusty" model atmospheres, Dr. D. Schwenke for $\mathrm{HDO}$ and $\mathrm{H}_{2} \mathrm{O}$ line lists. Partial support of my investigations was provided by Small Research Grant of AAS.

\section{References}

Basri, G., Martín, E.L. 1999. ApJ, 510, 266. (1999).

Béjar, V.J.S., Zapatero Osorio, M.R., Rebolo, R., 1999, ApJ, 521, 671.

Burrows, A., Marley, M., Hubbard, W.B.. 1997. et al. 1997. ApJ, 491, 856.

Chabrier, G., Baraffe, I., Allard, F., Hauschildt, P. 2000. å, 542, L122.

Hauschildt, P., Allard, F., Baron, E. 1999. ApJ, 512, 377.

Kupka, F., Piskunov, N., Ryabchikova, T. A., Stempels, H. C., Weiss, W. W. 1999. A\&A Suppl., 138, 119.

Martín, E.L., Basri, G., Gallegos, J.E. et al. 1998. ApJ, 499, L61.

Partridge, H., Schwenke, D. 1997. J. Chem. Phys., 106, 4618. 


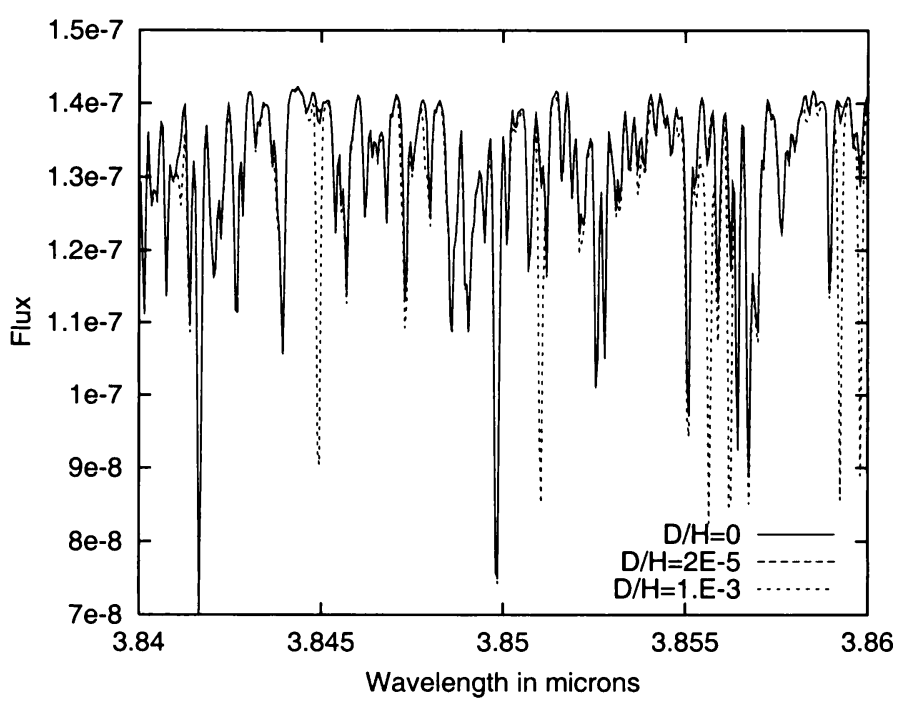

Figure 3. Theoretical spectra computed for different $\mathrm{D} / \mathrm{H}$ for region $3.84-3.86$ microns

Pavlenko, Ya.V., Rebolo, R., Martín, E.L., García López, R. 1995. Astron. Astrophys. 303, 807.

Pavlenko, Ya. V., 1997. Astrophys. Reports, 41, 537.

Pavlenko, Ya. V. 2000 Astron. Rept. 44, 219.

Pavlenko, Ya.V. 2002. Astron. Rept., 46, 567.

Rebolo, R., Martín, E.L., Magazzù, A. 1992. ApJ, 389, L83.

Rebolo, R., Martín, E.L., Basri, G., Marcy, G.W., Zapatero Osorio, M.R. 1996. ApJ469, L53.

Ruiz, M.T., S.K. Leggett, S.K., Allard, F. 1997. ApJ, 491, L107.

Saumon, D., Hubbart, W.B., Burrows, A., Gulliot, T., Lunine, J.I., Chabrier, G. 1996. ApJ,460, 999.

Stauffer, J., Schultz, G., Kirkpatrick, J.D. 1998. ApJ, 499, L199.

Tsuji, T. 2000. Very low mass stars and brown dwarfs, Eds. R. Rebolo, M. R. Zapatero Osorio. (Cambridge: Cambridge University Press), 126.

Zapatero Osorio, M.R., Béjar, V.J.S., Pavlenko, Ya., Rebolo, R., Allende Prieto, C., Martín, E.L., García López, R. 2002, A\&A, 384, 937. 